\title{
Tabla DK eLearning: optimización de la práctica docente en un ambiente online ${ }^{1}$
}

\section{DK eLearning grid: optimization of teaching practice in online environment}

\author{
Ana Maria Ferreira Nobre \\ Universidade Aberta, Lisboa, Portugal \\ ana.nobre@uab.pt \\ Daniela Melaré Vieira Barros \\ Universidade Aberta, Lisboa, Portugal \\ daniela.barros@uab.pt \\ Antonio Chenoll Mora \\ Universidade Católica Portuguesa, Lisboa, Portugal \\ a_chenoll@fch.lisboa.ucp.pt \\ Ana Setién Burgués \\ Universidade Aberta, Lisboa, Portugal \\ aburges@uab.pt
}

\begin{abstract}
Resumen
El presente trabajo es el resultado del estudio realizado en el ámbito de la práctica pedagógica en la enseñanza superior en e-learning. Observamos una mejora cualitativa y necesaria de las prácticas docentes online en sus modelos pedagógicos. El panorama teórico general en la educación enfatiza la necesidad de reforzar y perfeccionar las estructuras didácticas de la enseñanza online, pero existe una escasez de instrumentos de transposición didáctica para que eso suceda. En este escenario y con el objetivo de traer una contribución para el relleno de esa laguna, proponemos a través de elementos didáctico-pedagógicos y tecnológicos en contextos online, una tabla de observación con base al modelo de Amador et al. (2016)

Los procedimientos metodológicos fueron soportados por referencias bibliográficas, reflexiones y discusiones que acompañaron las diversas fases del estudio de la creación y validación de la tabla, Presentamos un ejemplo aplicado en el área de la lengua y cultura española y proponemos una estructura teórica como apoyo al desarrollo y sus resultados.

Palabras clave: Transposición didáctica; Didáctica del e-learning; Proceso de enseñanzaaprendizaje; Ambiente virtual; E-learning;
\end{abstract}

\begin{abstract}
The present work results from the study carried out in the scope of the pedagogical practice in higher education in e-learning. We observe a qualitative and necessary improvement of online and e-learning teaching practices in their pedagogical models, the general theoretical panorama in education emphasizes the need to reinforce and improve the didactic structures of online teaching, but there is a shortage of didactic transposition instruments for this to occur. In this scenario and with the objective of contributing to fill this gap, we propose through pedagogical and technological didactic elements in online and e-learning contexts, an observation grid based on the model, DK eLearning-TPCK of Amador et al. (2016). The methodological procedures were supported by bibliographic references, reflections and discussions that accompanied the various phases of the study in the creation and validation of grids. We present an example applied in the area of Spanish language and culture and
\end{abstract} propose a theoretical framework to support development and its results.

Keywords: Didactic transposition; Didactics of e-learning; Teaching-learning process;

Virtual environment; E-learning.

\footnotetext{
${ }^{1}$ Este estudio está realizado por un grupo de investigadores del Laboratório de Ensino a Distância e eLearning (LE@D), que son los autores del presente trabajo.
} 


\section{Introducción}

Mucho se ha hablado sobre el potencial transformador de la tecnología en la educación, pero ¿cómo orientar esa transformación en la clase? Para una mejor claridad sobre la cuestión Mishra e Koehler (2006) proponen el TPACK (Technological Pedagogical Content Knowledge) como un modelo que destaca la interacción entre el conocimiento pedagógico, el contenido y la tecnología. Amador, F., Nobre, A., Barros, D. (2016a) proponen una estructura basada en un tetraedro regular, el cual corresponde a un poliedro compuesto por cuatro caras triangulares (triángulo equilátero). Este modelo, que en adelante denominaremos DK eLearning-TPCK, se destaca principalmente de los otros modelos por el vértice/círculo central Didactic Knowledge en el aula virtual.

Una combinación eficiente para la integración de las tecnologías digitales en la educación depende de los conocimientos de contenido, pedagógico y tecnológico (Coutinho, 2011). Revisiones de la literatura apuntan a trabajos sobre la temática (Rosenberg y Koehler, 2015) que destacan esencialmente los aspectos pedagógicos y didácticos como interfaces y herramientas en ambientes online. Sin embargo, existe una carencia en la fundamentación teórica y sobre cómo las teorías de aprendizaje pueden servir de orientación para su desarrollo (Graham, 2011).

Para Mishra e Koehler (2006), la enseñanza con la tecnología es una actividad compleja pues recurre a las relaciones entre diferentes conocimientos. Para Amador Amador et al. (2016a) los elementos fundamentales del análisis (vértices Pedagogical Knowledge, Content Knowledge y Tecnological Knowledge) remiten a dominios transdisciplinares.

Partiendo de esos elementos, elaboramos un estudio realizado en la enseñanza superior portuguesa, en la modalidad de enseñanza a distancia e e-learning, en las licenciaturas de graduación y postgraduación y ejemplificamos en el área de lengua y cultura española. Con base al modelo de Amador et al. (2016a), analizamos la transposición didáctica en la enseñanza online, desarrollamos y visualizamos perspectivas que ayuden a la comprensión de este proceso. Con el objetivo de realizar una contribución para el relleno de esa laguna, identificamos los elementos didácticos pedagógicos y tecnológicos en contextos online, creamos y proponemos una tabla de observación del modelo DK eLearning-TPCK.

Fue realizada una investigación con aplicaciones, pruebas y discusión teórica científica sobre la temática y las cuestiones orientadas de este estudio son:

- Identificar los elementos didáctico-pedagógicos y tecnológicos en la enseñanza online.

- Verificar los procesos de transposición didáctica del conocimiento con el uso de las tecnologías.

- Contribuir a un autoanálisis en la modalidad de e-learning.

- Validar una tabla de observación para el área de la enseñanza de lenguas y culturas extranjeras.

Con estas cuestiones desarrollamos la propuesta aquí presentada.

\section{Contexto}

Presentamos el modelo DK elearning-TPCK, la enseñanza y el aprendizaje online y proponemos una estructura teórica como apoyo al desarrollo de una tabla de observación y sus resultados.

$\overline{\text { Tabla DK eLearning: optimización de la práctica docente en un ambiente online. A Nobre, D. Barros, A. }}$ 


\subsection{El modelo de transposición didáctica}

Uno de los impactos inherentes de la relación entre tecnología y educación es la integración de la tecnología en función de una acción docente actualizada y conducente con el desarrollo relacional del mundo. Relacionamiento este, maleable por una red cuya adaptación y flexibilidad solicitan la idiosincrasia evolutiva de las tecnologías de información y comunicación (Castells, 2004), y que inevitablemente, se extienden al espacio escolar. Como el trabajo del profesor en la clase está compuesto por una estructura de acción que envuelve saberes distintos y que una vez entrelazados, construyen el conocimiento como resultado de la praxis enseñanza-aprendizaje, deben emerger referenciales conceptuales y teóricos que fundamentan y reestructuren los procesos de enseñanza y aprendizaje sobre la incorporación de tecnologías en la educación.

La formación en tecnologías, vista como un vínculo de mediación entre tecnologías y saberes docentes (Maneira y Gomes, 2016) representados como competencias capaces de operacionalizar la cuestión de la competencia tecnológica (Wiebe y Taylor, 1997), es movilizadora de la actuación docente en el sentido contrario de las metodologías.

Las competencias docentes del siglo XXI divergen en recursos, tiempo y espacio en una dinámica de transformación; la manipulación de un instrumento digital permite innovación en el acto educativo, permeabiliza la dinámica pedagógica de las tecnologías, opera un nuevo sentido para la relación entre el profesor y alumno, principalmente en el proceso de enseñanza y aprendizaje.

Desde esta perspectiva, el referencial conceptual y teórico propuesto por Amador et al. (2016a) se figura particularmente adecuado en el repensar de la praxis (práctica/teoría/reflexión) docente. Así en nuestra lectura, el círculo central (Didáctica del e-learning - sala de aula virtual) está conectado a los tres vértices (PK, CK e TK) el tetraedro (ver figura 1), que funcionan integrados en el interior de otras dimensiones y se configuran como directrices conceptuales y teóricas en una perspectiva multidimensional, integrada e integradora, de los conocimientos (y competencias) que deben integrar la práctica pedagógica de los docentes. 


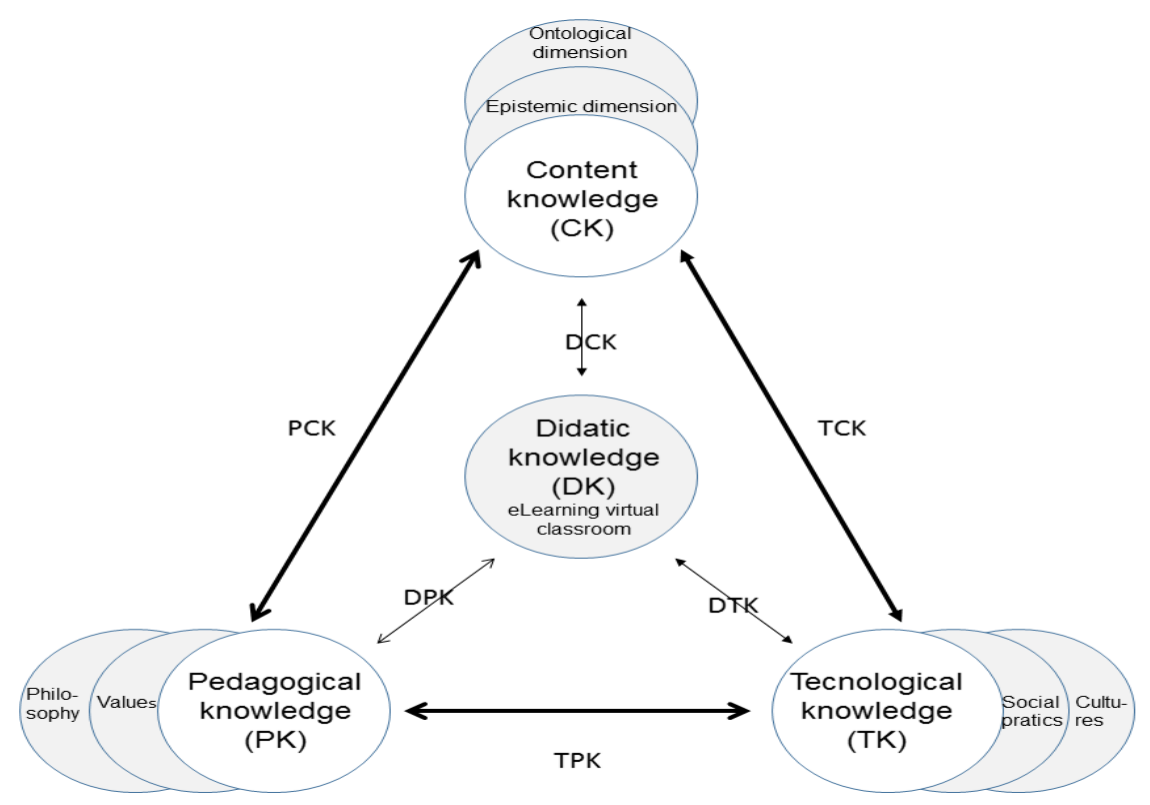
learning $^{2}$

Figura 1. Modelo de transposición didáctica, centrado en una didáctica del e-

En la figura 1, la letra «C» indica el conocimiento de contenido, el conocimiento conceptual del profesor y las respectivas dimensiones ontológicas y epistemológicas. De forma general, lo podemos definir como el contenido curricular del trabajo docente.

La letra «P» representa el Conocimiento Pedagógico, los valores y las filosofías. Cómo las estrategias pedagógicas del profesor se basan en teorías de aprendizaje y posibilitan el conocimiento de los (y a los) estudiantes, reconociéndolos al mismo tiempo en sus características y dificultades por medio de dinámicas de apelación.

$\mathrm{La}$ «T» se relaciona con el Conocimiento Tecnológico, a las prácticas sociales y culturales relacionadas con la adaptación de la tecnología tanto por parte del docente.

El quid de este modelo es la interdependencia entre los conocimientos didáctico, pedagógico, tecnológico y la enseñanza aprendizaje en la modalidad de la enseñanza distancia y e-learning.

\subsubsection{La transposición didáctica y la docencia.}

La intervención del profesor en ambientes online, el modelo de cinco etapas de Salmon (2008), sintetiza el papel del profesor en el proceso de construcción del conocimiento en el ambiente virtual de aprendizaje. El enfoque de "El aprendizaje situado" (Lave y Wenger, 1991) implica la participación en la comunidad de práctica, donde el aprendizaje no está considerado como un proceso de adquisición, sino de participación.

Este enfoque es indispensable para la construcción del pensamiento en el dominio y para la escenificación de la innovación y sustentabilidad de los modelos de aprendizaje colaborativo para la universidad en la sociedad digital (Tapscott y Williams 2010). Esta favorece la construcción de un marco de competencias en la concepción de organización de los aprendizajes colaborativos de los ambientes virtuales en la sociedad digital.

\footnotetext{
${ }^{2}$ En esta imagen, los autores mantuvieron la misma designación de las aristas en lengua inglesa.

Tabla DK eLearning: optimización de la práctica docente en un ambiente online. A Nobre, D. Barros, A. 
Las tecnologías digitales anuncian la necesidad de reconfiguración de la infraestructura (interna y externa) de las instituciones educativas y los profesores serán también orientadores con el objetivo de optimizar el pensamiento crítico, reflexivo y creativo de un estudiante más autónomo. Por tanto, las acciones pedagógicas exigen una mirada holística sobre las temáticas, acciones organizadas, fruto de la observación sobre el cómo, para qué, por qué y cuándo. Estás interrogaciones, en el contexto de la integración de las tecnologías digitales, pueden (y deben) ser articuladas con diferentes concepciones/diseños pedagógicos, pues cada una tiene algo que ofrecer, ya que necesitamos pensar en estructuras que optimizan el proceso de aprendizaje.

Cuando utilizamos las tecnologías, Skinner (1950) propone un condicionamiento a través de estímulos relacionados con la manipulación ordenada de las herramientas. En la Transposición Didáctica (en adelante TD), este proceso actúa como eje central: cómo el docente relaciona los temas con la realidad y cómo ofrece a los estudiantes un incentivo real con situaciones que motivan y estimulan el pensamiento.

La adaptación y organización propuesta por Jean Piaget (1969) proporciona al sujeto vía juegos y modelaje, experimentación empírica como forma de promover diferentes interacciones entre sujetos, objetos y situaciones. En el uso de las tecnologías la experimentación y la interacción en diferentes escenarios y contextos es una idea central, en la TD entendemos cómo la construcción de esos diferentes escenarios puede facilitar el marco de los contenidos para una mejor percepción de los estudiantes.

Gagné (1996a, 1996b), en su procesamiento de la información a partir de la selección de herramientas que motiven el aprendizaje y proporcionen la modificación y madurez resultantes, divide el aprendizaje en fases: la motivación, aprehensión, adquisición, retención, rememoración, generalización, actuación y retroalimentación. En la TD, pueden ser utilizadas en un proceso metodológico más directivo con estrategias y contenidos que atienden a los objetivos propuestos.

El construccionismo (Papert, 1980), gracias la reconfiguración de las condiciones de aprendizaje, intermediadas por las herramientas digitales ayuda a la organización del pensamiento por el sujeto. En la TD ayuda a construir estrategias apelativas y facilitan a los estudiantes instrumentos de asimilación para llegar al resultado final, o sea, el aprendizaje de contenidos.

Debido a este escenario epistemológico, a los nuevos diseños de enseñanza y aprendizaje posibles con uso de las tecnologías digitales en contexto educativo, debemos estimular la discusión y según Ausubel $(1968,1978)$ fomentar un aprendizaje significativo. Así, las tecnologías simulan estos saberes previos a partir de actividades que promueven el descubrimiento, o sea cuanto mayor el número de enlaces realizados, más consolidación habrá en el uso pedagógico. Es en esta conexión donde reside la fuerza de acción del docente, en saber cómo utilizar la tecnología a favor de procesos de enseñanza y de aprendizaje. Esta dinámica se visualiza y se presenta a través de una tabla de observación ${ }^{3}$ por docentes de Lengua y Cultura Española y por docentes observadores. Esta tabla enfatiza la interdependencia entre los conocimientos de contenido, pedagógicos y tecnológicos.

En este trabajo se discutirá la relación entre el referencial teórico del modelo propuesto por Amador et al. (2016a) y su conexión con la educación en la enseñanza superior. Se procuró comprender el modelo de formación implícito en la proposición del modelo DT centrado en una Didáctica del e-learning.

\footnotetext{
${ }^{3}$ Para acceder a la tabla puede dirigirse al siguiente enlace permanente: https://figshare.com/s/9dd5bf8d12f462cdb058
}

Tabla DK eLearning: optimización de la práctica docente en un ambiente online. A Nobre, D. Barros, A. 


\section{La enseñanza y el aprendizaje online de la lengua y la cultura.}

Investigadores en tecnología educativa postularon y repitieron que el interés pedagógico de una tecnología reside en la capacidad de implementar diversos modelos de aprendizaje. La lección fundamental no es optar por una tecnología específica, sino definir una secuencia o un ambiente de aprendizaje de acuerdo con el modelo pedagógico que responda a las expectativas de los estudiantes.

$\mathrm{Si}$ aceptamos ese paradigma de preponderancia y complementariedad del modelo pedagógico sobre la tecnología, es a través de sus modelos pedagógicos y de su evolución con las que renovamos las prácticas educativas.

Uno de los principales temas de la enseñanza a distancia es el papel central desempeñado por el estudiante en el proceso de construcción, desarrollo y perfeccionamiento de su propio conocimiento. El docente, como experto en procesos de adquisición, ha de interrogarse con la mayor objetividad posible sobre cuál es el objetivo de nuestras clases; para qué puede servirle estos contenidos al alumno; cómo aprende contenidos o cuál es la influencia de nuestro filtro cognitivo en la manera de percibir, organizar y producir contenido.

Desde esta perspectiva, y de la misma manera que el aprendizaje de contenidos explícitos, la adquisición de lenguas extranjeras (desarrollo de capacidades implícitas) ha enfocado sus medidas de actuación en, al menos, cuatro niveles: El alumno como centro del proceso, la acción como fin y medio del aprendizaje, la autonomía e independencia en el aprendizaje y, finalmente, la adaptación al espectro cognitivo individual del alumno.

El papel del docente, por tanto, debe adaptarse a esta perspectiva del alumno activo, debe ser un papel de gestor de esa información en función del espectro cognitivo del alumno (Peris, 1998). Es un condicionante clave en el proceso de aprendizaje (Barbasán, 2016; Martín, 2000). El docente que no tenga en cuenta y no adapte la metodología de su asignatura a esta característica del aprendiente, no podrá optimizar el proceso de adquisición.

Por otra parte, somos conscientes de la importancia del desarrollo de la autonomía en el aprendizaje (Flores y Meléndez, 2017; García Salinas, Ferreira Cabrera, y Morales Rios, 2012). Desde nuestra perspectiva, uno de los objetivos fundamentales en el sistema universitario actual es el de enseñar no solo contenido, sino también las herramientas para que puedan conocer cómo aprenden mejor. Para ello, el papel del docente es fundamental. El docente no solo tiene que ser un experto en la materia que está impartiendo, además debe poseer conocimientos didácticos sobre los procesos de enseñanza y aprendizaje. Conocimientos basados en reflexiones serias y datos que nos ayuden a conocer objetivamente cómo aprendemos. Esto es, evitar confundir la subjetivación cualitativa por la objetivación cuantitativa. El modelo cuanti-cualitativo resultará el más adecuado para este menester. En definitiva, cuando finaliza la unidad curricular, acaban las notas y los trabajos con fecha; pero no el proceso de aprendizaje. Esto es, acaba el proceso de enseñanza; pero no el de aprendizaje.

Como hemos visto, consideramos que el aprendizaje online es inevitable. De la misma manera, que, siendo un elemento activo de la sociedad, es inevitable mantenerse al margen de la digitalización. Es inevitable, a su vez, un aprendizaje optimizado fuera de cualquier medio digital. Esto es así incluso desde la aparición de los vinilos aplicados a la enseñanza de lenguas (analógicos aún) y continuando con los casetes (ya digitales) que popularizaron las grabaciones y la tecnología para el aprendizaje de lenguas ambos desde una perspectiva audiolingual (Richards y Rodgers, 1986).

$\overline{\text { Tabla DK eLearning: optimización de la práctica docente en un ambiente online. A Nobre, D. Barros, A. }}$ 
Es importante resaltar que, de la misma manera que cualquier aprendizaje de contenidos, el objetivo del aprendizaje formal online es el de facilitar al alumno la reflexión sobre los contenidos con el propósito no solo de que el alumno, al reflexionar, cree una marca personal en el aprendizaje que le ayude a memorizar los mismos, sino que además le ayude a realizar una transferencia de esos mismos contenidos a otros ámbitos comunes.

Desde el punto de vista del aprendizaje online, como hemos podido observar partimos de una base en la que el aprendizaje optimizado, en un contexto universitario, no puede ser desconectado de la tecnología. De alguna manera, el aprendizaje formal está inevitablemente conectado con el aprendizaje digital y en red. Debemos (docentes online) encontrar prácticas pedagógicas alternativas para minimizar el impacto de algunas lagunas (el componente afectivo) y proporcionar al alumno soluciones que, sin ignorar el carácter masivo y asíncrono que muchos alumnos exigen, podamos adecuarnos a las necesidades de aquellos cuyo modelo de aprendizaje está más ligado al modelo presencial que a las prácticas autónomas e independientes del aprendizaje online.

En resumen, el aprendizaje debe estar supeditado al enfoque basado en la acción como medio y como fin, así como a la adecuación realística al perfil cognitivo y social del alumno y del grupo.

\section{Metodología}

Las opciones metodológicas de este estudio se basan en una significativa diversidad de estrategias de recogida de información, privilegiando el recurso a metodologías cualitativas teniendo en cuenta el objeto de estudio.

No optamos por denominar el trabajo desarrollado como "estudio de caso" ya que para Yin (2005) esta tipología es un enfoque metodológico de investigación en que queremos comprender, explorar o describir sucesos y contextos complejos en los cuales están simultáneamente envueltos diversos factores. Sin embargo, debido a la dimensión del trabajo que limita la introducción de elementos de información complementarios que podrían conducir a procesos de triangulación de datos, así como por una mayor contextualización de los estudios, se optó por designarlo como estudio para la construcción de un instrumento de observación e identificación de elementos didácticos pedagógicos.

La realización del estudio tuvo como sustento teórico las referencias del modelo pedagógico de la Universidade Aberta (Pereira et al., 2007) y demás fundamentos que sustentan los aspectos pedagógicos de la educación a distancia como: Rudestan y ReadSchoenholt (2010), Garrison (1989), Aretio et al (2007), Anderson (2005); Garrison y Anderson (2003); Downes (2007) e Peters (2001).

Como métodos de recolección y análisis de datos seleccionamos la observación no participante. La utilización de esta tipología de observación como medio de entrar en el mundo específico del contexto de los estudios organizacionales tiene una importancia creciente. Una de las razones es que no exista otra forma de que el investigador consiga acceso a determinado campo. Otra consideración del método recae sobre la posibilidad de que el investigador aprenda más sobre las actividades de las personas en su contexto natural (Kawulich, 2005), así como, la posibilidad de que el investigador observe y, en cierta forma, experimente, no solamente observando lo que está sucediendo, sino también sintiendo (Saunders, Lewis y Thornhill, 2000). 


\subsection{Diseño de la investigación}

Para construir la tabla de observación DK eLearning-TPCK, partimos del referente de Amador et al. (2016a) en su estructura didáctico-pedagógica englobando los elementos del TPCK. En este estudio, la observación de espacios de enseñanza y aprendizaje únicamente online constituye un proceso colaborativo entre el docente y su concepción didáctico-pedagógica, para asegurar beneficios mutuos en el desarrollo personal y profesional (Reis, 1999).

Según Vieira y Moreira (2011) existen ciclos de observación compuestos por tres fases principales, o sea, la pre-observación, observación y pos-observación. Estos ciclos están adaptados a la educación presencial, en la educación a distancia podemos estructurar estas fases de observación considerando la siguiente estructura: preobservación, análisis de factores contextuales, nivel y ciclo de estudios, enfoques, modelos pedagógicos y materiales disponibles. La observación propiamente dicha es la recolección de la información en función de los objetivos predefinidos. Por su parte, la pos-observación es el análisis y la retroalimentación informativa a través de la confrontación de teorías, fundamentos y prácticas existentes.

Considerando estas afirmaciones, la estructura del diseño de investigación fue establecida a partir de la construcción del instrumento de observación, la observación realizada, los resultados y el análisis a partir de elementos del modelo propuesto por Amador et al. (2016a), resultando en la construcción del instrumento de orientación pedagógica y, por tanto, en la construcción de cursos online de enseñanza de lengua y de cultura

En resumen, el diseño del estudio realizado puede ser visualizado de la siguiente forma.

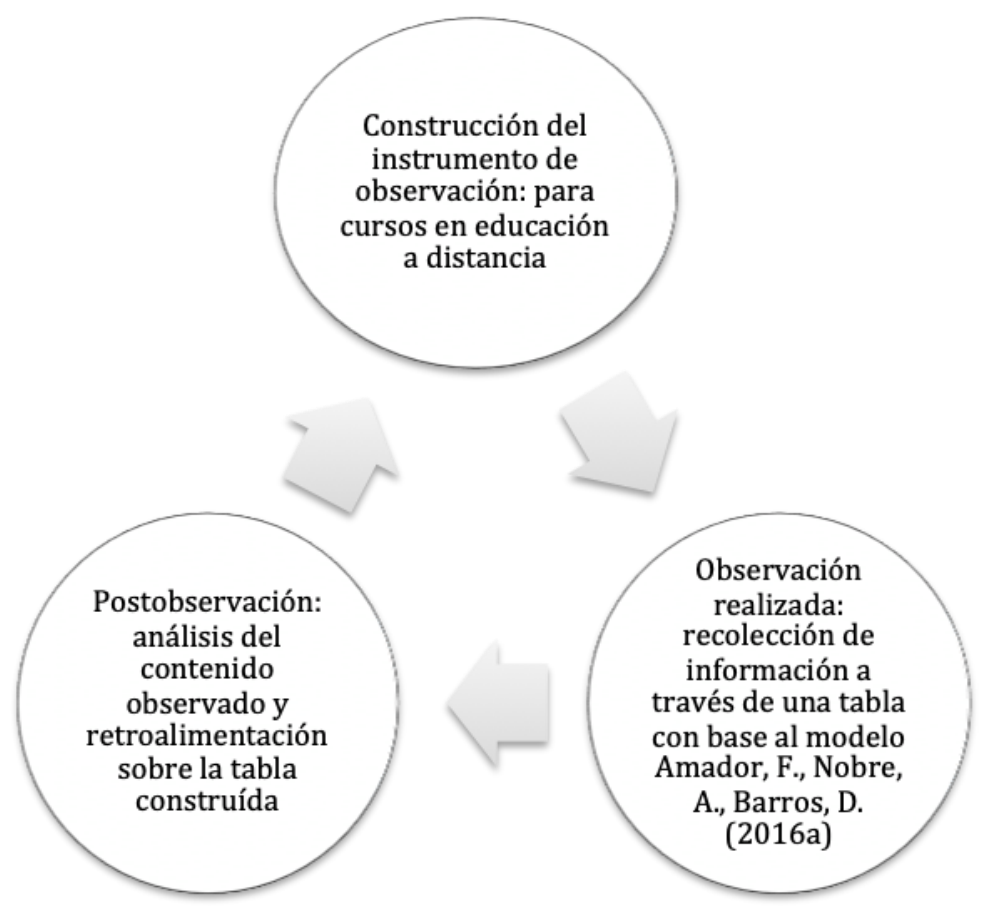

Figura 2: Diseño metodológico del estudio realizado.

\subsection{Etapas para la construcción de la tabla de observación.}

A partir del diseño de investigación definido se describen las etapas (Figura 3)

Tabla DK eLearning: optimización de la práctica docente en un ambiente online. A Nobre, D. Barros, A. 
siguientes para la construcción del instrumento. Estas etapas fueron desarrolladas a lo largo de seis meses y son las siguientes:

Búsqueda bibliográfica en bancos de datos nacionales e internacionales

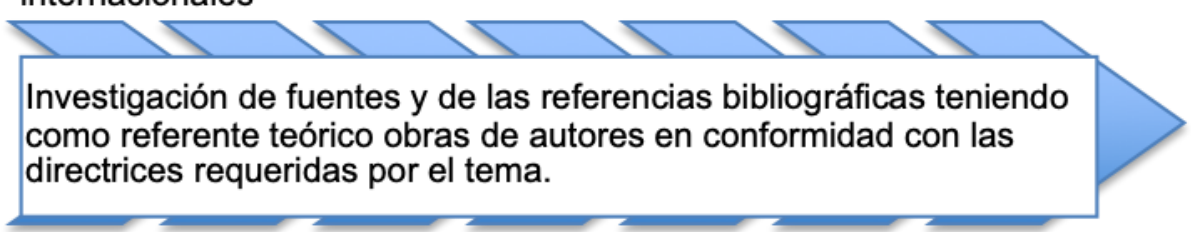

Definición de parámetros de avaluación a través de la observación no participante.

La inexistencia de una escala u otra herramienta que pudiese ser
adaptada a la didáctica del elearning fue construido un instrumento
propio para este fin. Se realizaron sesiones de discusión con
especialistas en el área de la educación.

Elaboración de los ítems de la tabla.
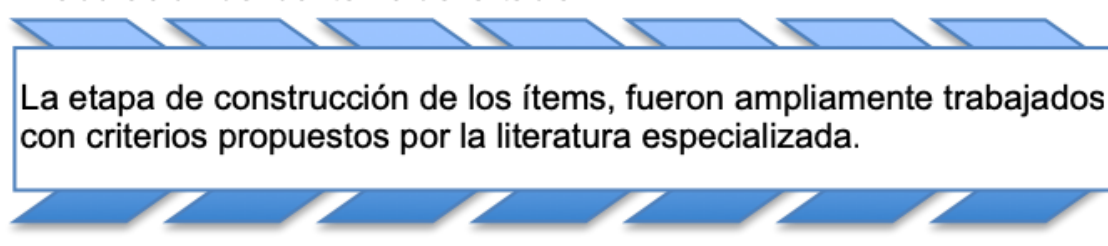

Definición de la escala de observación

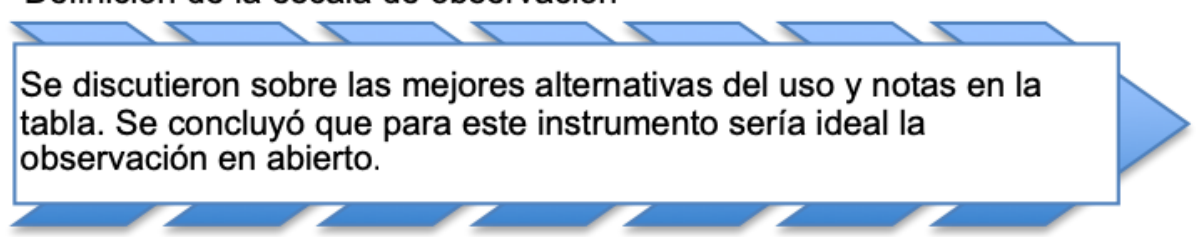

Elaboración de la primera versión de la tabla.

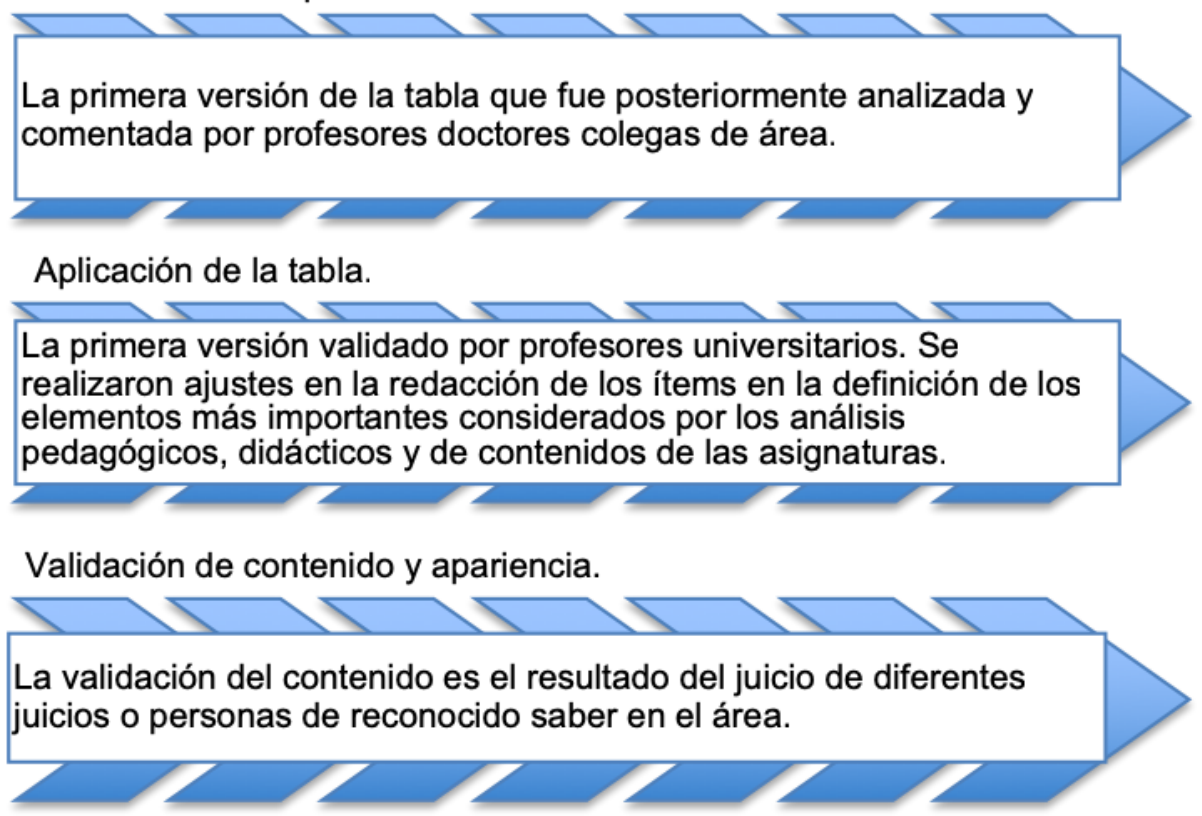

Figura 3: Etapas de elaboración e descripción de las acciones

$\overline{\text { Tabla DK eLearning: optimización de la práctica docente en un ambiente online. A Nobre, D. Barros, A. }}$ 
Se sometió la tabla DK - Learning a una evaluación de los profesores/investigadores de la Universidade Aberta. De esta forma, la idea fue realizar una validación del contenido por los colegas investigadores, buscando determinar la apropiación de los ítems y dimensiones del instrumento. La validación del contenido no está determinada estadísticamente, pero estos analizan la representatividad de los ítems en relación a los conceptos evaluados (Anastasi \& Urbina, 2000; Fachel \& Camey, 2000; Pasquali, 2001). Para estos autores, el instrumento posee validad de contenidos y los ítems que lo constituyen son representativos del universo que él pretende representar y además de eso sí son, desde el punto de vista teórico, indicadores de la variable a ser medida. Además de la validez del contenido propiamente dicho, encontramos la validad de cara o validad aparente que se refiere a lo que el test mide superficialmente y se refiere a la lengua y a la forma en como el contenido está siendo presentado (Latiesa, 1996). Los pareceres de esos colegas indicaron que el instrumento era adecuado a los objetivos que se proponía, siendo el formato de la escala y la formulación de los ítems elementos facilitadores para su comprensión y relleno.

\subsection{Público objeto, espacio de investigación y procedimientos.}

En el desarrollo de la tabla de observación, como mencionado en las etapas arriba descritas, dos docentes de la Universidade Aberta, Portugal utilizaron las tablas en dos Unidades Curriculares (UC). Esas UCs eran, una, de enseñanza de lengua española y, otra, sobre Cultura Española ambas con graduación.

Los cursos eran totalmente online y asíncronos (plataforma Moodle) dentro del Modelo Pedagógico Virtual de la Universidade Aberta. La observación sucedió en un primer semestre de 2017 y 2018 e individualmente.

\section{4 tabla de observación.}

La tabla de observación elaborada por los investigadores contó con los siguientes elementos como objetivos de observación: identificar los aspectos pedagógicos de los cursos en e-learning; inventariar los principales errores didácticopedagógicas en las estructuras de los cursos facilitando la mejoría de estas estructuras en la enseñanza y aprendizaje online.

Las dimensiones de la tabla fueron: conocimiento didáctico; pedagógico; tecnológico y de contenido. Esos elementos son comunes al desarrollo de cursos online como podemos identificar en Pereira et al. (2007) y demás fundamentos que sustentan los aspectos pedagógicos del eLearning como: Rudestan y Read-Schoenholt (2010), Garrison (1989), Aretio et al. (2007), Anderson, (2005), Garrison y Anderson (2003), Downes (2007).

La tabla DK - eLearning elaborada en Excel calcula automáticamente los valores estipulados en una escala de 1 a 5 siendo 1 el mínio y 5 el máximo para cada uno de los puntos observar. Existen algunos ítems que tienen valores multiplicadores por considerarse de más importancia en el proceso de evaluación. Los resultados de sus valores sumados al final y representados en porcentajes representan un gráfico, un tetraedro que resalta los resultados finales para cada dimensión como se puede observar en la figura 4. 


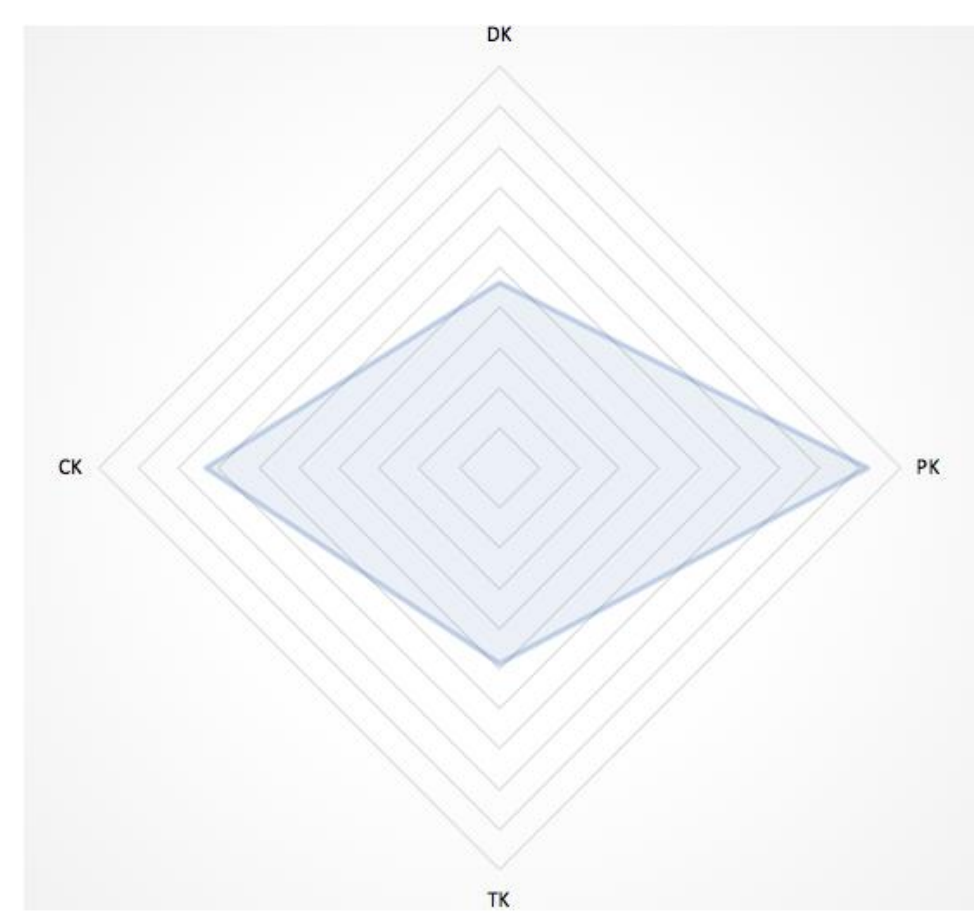

Figura 4: Ejemplo de resultados de la transposición didáctica en la asignatura de Sociedade e cultura Espanhola

El tratamiento de los datos recogidos en la observación utilizó el análisis de contenido, anotaciones y elementos identificados por los investigadores. Seguidamente describiremos los resultados obtenidos en la prueba de la tabla.

\section{Resultado y discusiones}

Como parece evidente, el análisis de la práctica docente es siempre un elemento arriesgado ya que depende, en gran medida, de la visión y creencias que se tenga sobre este. Con todo, es nuestra consideración es que la construcción mental que realizamos de nuestra UC es una ilusión (en el sentido cognitivo de la palabra) que puede acercarse más o menos a la realidad de los otros agentes implicados en el proceso. Es por eso mismo, un instrumento de objetivación sobre nuestras prácticas deberá siempre ser un eje fundamental en la evaluación que tienda a la objetividad de esta.

En el proceso de transposición didáctica hemos comprobado cómo es posible identificar los elementos con menos consideración para definir un plano de intervención que los considere por igual dentro del proceso de aprendizaje. No obstante, no se ha pretendido señalar que el CK es valorado más o menos que el TK o el PK, por ejemplo, ya que esa decisión formará parte de las consideraciones de cada docente; sino que el objetivo es marcar en qué aspectos el docente ha otorgado menor importancia para que, si lo considera oportuno, pueda intervenir de manera adecuada y minimizar el impacto de esta descompensación.

Por otra parte, se ha observado que cada UC tiene características especiales que se deben tratar de manera diferente y adecuado a las necesidades de los objetivos didácticos de cada disciplina. En las UC analizadas (lengua y cultura española) podemos observar que la UC de lengua necesita un componente mayor de producción libre y propia con el objetivo de consolidar los aprendizajes. En otras palabras, una UC de lengua extranjera exige del alumno la puesta en práctica de los contenidos con el objetivo de adquirir competencias en esa lengua. De manera diferente, una UC como 
Sociedad y Cultura Española, exige del alumno una mayor capacidad de reflexión y de conexión con otros contenidos explícitos.

Es exactamente por este motivo que la tabla no es un elemento pétreo, sino más bien un punto de partida, modificable, que deberá adecuarse a las exigencias de cada disciplina, sea esta más caracterizada por contenidos explícitos (historia, ciencias naturales, cultura), implícitos (lenguas maternas y extranjeras) o abstractos (matemáticas, ciencias exactas).

Sin embargo, aun con todas estas diferencias idiosincrásicas que se observan en las exigencias de cada disciplina, podemos considerar que todas pueden ser objeto del análisis de la transposición didáctica al contexto online.

En definitiva, el resultado del uso del análisis de la transposición didáctica nos ayudará a tomar una posición de alteridad sobre el producto que nosotros hemos creado para ayudarnos a la adaptación de los contenidos en un ambiente online de la manera más adecuada a nuestros objetivos y el de nuestros alumnos.

\section{Consideraciones finales y conclusiones}

Como hemos podido comprobar, los elementos que componen la transposición didáctica revisados a través de una tabla en la que se discriminan los elementos más importantes para tener en cuenta, nos ofrece una perspectiva evaluadora y visual de los puntos fuertes y los puntos débiles de nuestra práctica docente.

Esta herramienta servirá tanto a los docentes responsables y alumnos, como a elementos externos a la asignatura para medir e identificar de manera cuantitativa y cualitativa los elementos que deben ser reforzados para conseguir tener una UC compensada y en la que se consideren todos los elementos de manera objetiva.

Este hecho se reviste de una especial importancia cuando el objeto de estudio sucede en un contexto completamente a distancia y online en el que, de manera general, suele otorgarse más importancia al contenido que a la pedagogía y didáctica de esos mismos contenidos. Sabemos que el contexto de enseñanza a distancia y online, posee una idiosincrasia especial desde el punto de vista de la manera en cómo los contenidos son presentados, en la relación con las prácticas de estudio y reflexión con alumnos e incluso se perciben claras diferencias en el componente afectivo de los elementos envueltos en el proceso de enseñanza y aprendizaje. Este tipo de prácticas críticas nos ayudarán a minimizar las posibles lagunas en las que, tal vez involuntariamente, los docentes hayan podido incurrir.

En este sentido, en ningún caso debemos considerar los resultados de la tabla de observación como siendo un dictamen exacto de las características de la UC; sino más bien, una tendencia que nos abre una puerta a la reflexión, a la crítica constructiva y a una optimización de la práctica docente en un ambiente online y virtual.

En definitiva, consideramos que el uso de esta tabla supone un empoderamiento del proceso de enseñanza y aprendizaje por parte de los interventores de este y promueve una reflexión lógica y con datos objetivos sobre las características reales de nuestra práctica docente en un ambiente online.

Algunas conclusiones las podemos resumir en las siguientes:

i.Es necesario un producto de reflexión sobre nuestra práctica docente online.

ii.Es necesario ser consciente de que el aprendizaje online posee características particulares a las que le debemos otorgar la importancia necesaria.

iii.Debemos adaptar la transposición didáctica online según el tipo y objetivo de la disciplina, así como a sus exigencias cognitivas.

$\overline{\text { Tabla DK eLearning: optimización de la práctica docente en un ambiente online. A Nobre, D. Barros, A. }}$ 
iv.El uso de una tabla cualitativa y cuantitativa nos ayuda a delimitar las descompensaciones habidas en la práctica docente online.

El proceso de enseñanza y aprendizaje es un proceso excepcionalmente interesante no solo por las preguntas y retos que plantea a quien debe diseñar un proceso optimizador, sino porque nos ayuda a entender la realidad, tal como la visión del aprendizaje, como una construcción basada en axiomas los cuales definen qué vemos y qué queremos ver. La importancia que le otorgamos a cada elemento de la educación (y de la realidad) viene definido por nosotros mismos. Si exigimos de nuestros alumnos una visión no sesgada de la realidad, deberemos también realizar un ejercicio crítico de nuestra práctica docente online, tener la valentía de saber rectificar y hacer de nosotros unos mejores guías del aprendizaje.

Presentación del artículo: 30 de enero de 2019 Fecha de aprobación: 6 de marzo de 2019 Fecha de publicación: 30 de abril de 2019

Nobre, A., Barros, D., Chenoll, A., Setién, A. (2019). Tabla DK eLearning: optimización de la práctica docente en un ambiente online. RED. Revista de Educación a Distancia, 59. DOI http://dx.doi.org/10.6018/red/59/03

\section{Financiación}

Esta investigación no ha recibido ninguna subvención específica de los organismos de financiación en los sectores públicos, comerciales o sin fines de lucro.

\section{Referencias bibliográficas:}

Amador, F., Nobre, A., Barros, D. (2016a). "Towards a model of a didactic of eLearning: an application to education for sustainable development", Handbook of Research on Engaging Digital Natives in Higher Education Settings, IGI GLOBAL. DOI: 10.4018/978-1-5225-0039-1.ch019.

Amador, F.; Nobre, A.; Barros, D. ;Albuquerque, L. G.- A transposição didática no ámbito do eLearning In. DIas,P.:Moreira,D.:;Quintas-Mendes,A. (2016) Práticas e Cenários de Inovação em Educação Online. Universidade Aberta,Lisboa, Portugal, ISBN 9789726747864

Anastasi, A. Urbina, B. S. (2000). Testagem psicológica. Porto Alegre: ArtMed.

Anderson, T. (2005). Distance learning - Social software's killer ap? 17th Biennial Conference of the Open and Distance Learning Association of Australia (ODLAA).

Anderson, T. ( 2008) Theory and Practice of Online Leanring, $2^{\mathrm{a}}$ ed. AU Press, Athabasca University.

Aretio, L. G., Corbella, M. R., Figaredo, D. D. (2007). De la educación a distancia a la educación virtual. Barcelona: Editorial Ariel.

AusubeL, D.P. ( 1968) Educational Psychology: A Cognitive View. New York, Holt, Rinehart and Winston.

Ausubel, D., Novak, J., Hanesian, H. (1978), Educational Psychology: A Cognitive View ( $2^{\mathrm{d}}$ Ed.), New York: Holt, Rinehart \& Winston.

$\overline{\text { Tabla DK eLearning: optimización de la práctica docente en un ambiente online. A Nobre, D. Barros, A. }}$ 
Bandura, A. (1987). Pensamiento y Acción: Fundamentos Sociales. Barcelona: Ediciones Martínez Roca.

Barbasán Ortuño, I. P. (2016). El error léxico en la interlengua de lenguas afines: italiano y español.

Bruner, J. (1960). The Process of Education. Cambridge. Harvard University Press.

Castells, M. (2004). A Galáxia da Internet: Reflexões sobre Internet, Negócios e Sociedade. Lisboa: Fundação Calouste Gulbenkian

Coutinho, C. P. (2011). TPACK : em busca de um referencial teórico para a formação de professores em Tecnologia Educativa.Revista Paidéi@, vol.2(n.4), 18.

Downes, S. (2007). e-Learning 2.0 in development. Brandon Hall Research Innovations in Learning Conference, San Jose, California. Áudio e slides disponíveis em http://www.downes.ca/presentation/149

Fachel, J. Camey, S. (2000). Avaliação psicométrica: a qualidade das medidas e o entendimento dos dados (pp. 158-170). In: J. A. Cunha e cols. Psicodiagnóstico. (v. 5, ed. rev.) Porto Alegre: Artmed.

Flores, L. D., Meléndez, C. F. (2017). Variación de la autonomía en el aprendizaje, en función de la gestión del conocimiento, para disminuir en los alumnos los efectos del aislamiento. Revista De Educación a Distancia (RED), (54).

Gagné, R. M. (1996a). Learning processes and instruction. Training Research Journal. $1(1), p 17-28$.

Gagné, R. M. Medsker, K. (1996). The conditions of learning: Training Applications. Fort Worth, TX: Harcourt Brace College Publishers.

García Salinas, J., Ferreira Cabrera, A., Morales Rios, S. (2012). Autonomía en el aprendizaje de lenguas extranjeras en contextos de enseñanza mediatizados por la tecnología. Onomázein, 1(25).

Garrison, D. Randy (1989). Understanding Distance Education - a framework for the future. Routledge: London and New York, p. 139.

Garrison, R. Anderson, T. (2003). eLearning in the 21st Century: A Framework for Research and Practice. London \& New York: RoutledgeFalmer.

Kawulich, B. B. (2005). Participant observation as a data collection method. Forum: Qualitative Social Research [On-line journal]. v. 6, n. 2, art. 43.

Kostoulas, A., Stelma, J., Lee, W., \& Onat-Stelma, Z. (2015). Intentional Dynamics in TESOL: An Ecological Perspective. Working Papers in TESOL and Applied Linguistics, 15.

Kumaravadivelu, B. (1994). The Postmethod Condition: (E)merging Strategies for Second/Foreign Language Teaching. TESOL Quarterly, 28(1), 27.

Kumaravadivelu, B. (2001). Toward a Postmethod Pedagogy. TESOL Quarterly, 35(4), 537-560.

Latiesa, M. (1996). Validez e fiabilidad de las observaciones sociologicas. Em F. M. Garcia, J. Ibáñez F. Alvira (1996). El analisis de la realidad social: métodos e técnicas de investigación. 2a ed. (pp. 335-364). Madri: Alianza.

Lave, J.; Wenger, E. (1991) Situated Learning: Legitimate peripheral participation. Cambridge: Cambridge University Press.

Lewis, M. (1993). The lexical approach: The state of ELT and the way forward. Hove, England. Language Teaching Publications.

Lewis, M. (1997). Implementing the lexical approach: Putting theory into practice. Hove, England. Language Teaching Publications.

Maneira, S., Gomes, M. J. (2016). Professores e TPACK: Uma revisão sistemática da literatura. In L. Miranda, P. Alves, C. Morais (Eds.), Livro de Atas do VII Congresso Mundial de Estilos de Aprendizagem (pp. 1345-1360). Bragança:

$\overline{\text { Tabla DK eLearning: optimización de la práctica docente en un ambiente online. A Nobre, D. Barros, A. }}$ 
Instituto Politécnico de Bragança.

Martín Martín, J. M. (2000). La lengua materna en el aprendizaje de una segunda lengua. Sevilla. Universidad de Sevilla.

Mishra, P., Koehler, M. J. (2006). Technological pedagogical content knowledge: A framework for teacher knowledge. Teachers college record, 108(6), 1017 - 1054. Papert, S. (1993). Children's machine: Rethinking school in the age of the computer. New York: Basic Books.

Morais, C. ( 2005) Escalas de Medida, Estatística Descritiva e Inferência Estatística

Papert, S. M.(1980) Mindstorms: Children, Computers and Powerful Ideas, New York: Basic Books, 1980.

Pasquali, L. (1996). Teoria e métodos de medida em ciências do comportamento. Brasília: Laboratório de Pesquisa em Avaliação e Medida da Universidade de Brasília.

Pereira, A.; Quintas Mendes, A.; Morgado, L.; Amante, L.; Bidarra, J. (2007) Modelo Pedagógico Virtual da Universidade Aberta, Lisboa, Universidade Aberta.

Peris, E. M. (1998). El profesor de lenguas: papel y funciones. Conceptos clave en didáctica de la lengua y la literatura, 1998, págs. 87-100 (pp. 87-100). Institut de Ciències de l'Educació, ICE.

Peters, O ( 2001). Didática do ensino a distañcia: experiências e estágio da discussão numa visao internacional. Traduação Ilson Kayser. São leopoldo, Unisinos.

Piaget, J. (1969). Psychologie et pédagogie, Denoel, Paris, 264p

Reis, F. A. G. (1999). Avaliação do Desempenho e Identidade Profissional Docente. Dissertação de Mestrado em Educação. Faculdade de Psicologia e Ciências de Educação. Universidade do Porto.

Richards, J. C., Rodgers, T. S. (1986). Approaches and Methods in Language Teaching. Cambridge: Cambridge University Press.

Rosenberg, J. M., Koehler, M. J. (2015). Context and Technological Pedagogical Content Knowledge (TPACK): A Systematic Review. Journal of Research on Technology in Education, 47(3), 186-210.

Rudestan,K.E; Read-Schoenholtz. J. (2010). Handbook of online Learning. SAGE: Unites Estates of American.

Saunders, M. N. K.; Lewis, P.; Thornhill, A. (2000). Research methods for business. Pearson: England.

Skinner, B. F.(1950) Are theories of learning necessary? The Psychological Review, 57, pp. 193- 216.

Tapscott, Don; Williams, Anthony D. ( 2010) The Prosumers. In: Wikinomics: how mass collaboration changes everything. New York, USA: Penguin Books,

Vieira, F. Moreira, M.A. (2011).Supervisão e avaliação do desempenho docente. Para uma abordagem de orientação transformadora. Conselho científico para a avaliação de professores. Ministério da educação

Vygotsky, L. (1978). Interaction between learning and development.Readings on the development of children, 23(3), 34-41.

Wiebe, J. H., Taylor, H. G. (1997). What should teachers know about technology? A revised look at the ISTE foundations. Journal of Computing in Teacher Education, 13(3), 5-9

Yin. R. K. (2005). Estudo de caso: planejamento e métodos. 3 ed., Porto Alegre: Bookman 